\title{
Free fatty acid-induced histone acetyltransferase activity accelerates lipid accumulation in HepG2 cells
}

\author{
Sangwon Chung ${ }^{1}$, Jin-Taek Hwang ${ }^{1,2}$, Jae Ho Park ${ }^{1}$ and Hyo-Kyoung Choi ${ }^{1,5}$ \\ ${ }^{1}$ Korea Food Research Institute, 245, Nongsaengmyeong-ro, Iseo-myeon, Wanju-gun, Jeonbuk 55365, Republic of Korea \\ ${ }^{2}$ Department of Food Biotechnology, Korea University of Science \& Technology, Daejeon 34113, Republic of Korea
}

BACKGROUND/OBJECTIVES: Non-alcoholic fatty liver disease (NAFLD) is a common metabolic disease triggered by epigenetic alterations, including lysine acetylation at histone or non-histone proteins, affecting the stability or transcription of lipogenic genes. Although various natural dietary compounds have anti-lipogenic effects, their effects on the acetylation status and lipid metabolism in the liver have not been thoroughly investigated.

MATERIALS/METHODS: Following oleic-palmitic acid (OPA)-induced lipid accumulation in HepG2 cells, the acetylation status of histone and non-histone proteins, HAT activity, and mRNA expression of representative lipogenic genes, including PPARY, SREBP-1C, ACLY, and FASN, were evaluated. Furthermore, correlations between lipid accumulation and HAT activity for 22 representative natural food extracts (NExs) were evaluated.

RESULTS: Non-histone protein acetylation increased following OPA treatment and the acetylation of histones H3K9, H4K8, and H4K16 was accelerated, accompanied by an increase in HAT activity. OPA-induced increases in the mRNA expression of lipogenic genes were down-regulated by C-646, a p300/CBP-specific inhibitor. Finally, we detected a positive correlation between HAT activity and lipid accumulation (Pearson's correlation coefficient $=0.604$ ) using 22 NExs.

CONCLUSIONS: Our results suggest that NExs have novel applications as nutraceutical agents with HAT inhibitor activity for the prevention and treatment of NAFLD.

Nutrition Research and Practice 2019;13(3):196-204; https://doi.org/10.4162/nrp.2019.13.3.196; plSSN 1976-1457 elSSN 2005-6168

Keywords: Histone acetyltransferases, lipogenesis, non-alcoholic fatty liver disease, lipid metabolism, HepG2 cell

\section{INTRODUCTION}

Non-alcoholic fatty liver disease (NAFLD) associated with excessive lipid accumulation in the liver is the most common cause of chronic liver diseases, including obesity, dyslipidemia, hypertension, insulin resistance, and diabetes [1]. At the molecular level, NAFLD involves epigenetic alterations in the liver [2]. Lysine (Lys) acetylation is a crucial post-translational modification for the epigenetic regulation of genes. At least 2000 proteins in human cells can be post-translationally modified at lysine residues via acetylation [3,4]. Nearly 65 percent of these Lys-acetylated proteins are correlated with metabolism [5-7]. Thus, the dysfunction of Lys acetylation is major cause of metabolic syndrome, including obesity, cardiovascular disease, hypertension, type 2 diabetes, dementia, cancer, and NAFLD $[8,9]$.

Two important families of enzymes, histone acetyltransferases (HATs) and histone deacetylases (HDACs), coordinate the steady state of acetylation [10]. HATs add acetyl groups to the Lys $\varepsilon$ -amino residues of histones besides other non-histone proteins; in contrast, HDACs remove acetyl groups [11,12]. To respond to changes in metabolic demands and energy flux, metabolic enzymes (non-histone proteins) alters their catalytic activity or stability through the acetylation $[13,14]$. With respect to the regulation of metabolic enzymes in the liver, the balance of histone acetylation/deacetylation by HATs and HDACs is important.

NAFLD is conventionally explained by a "two-hit" model. Hepatic lipid accumulation is the first hit, causing insulin resistance, and excess inflammation by inflammatory cytokine interactions acts as the second hit [2]. However, recently, a new model in which multiple parallel hits are responsible for the development of NAFLD has been described [15]. The p300 HAT enzyme, which functions as a transcriptional coactivator, is involved in the first hit and second hit. It is co-recruited with nuclear factor- $\kappa \mathrm{B}$ to regulate various inflammatory signaling pathways [16] and increases the transcriptional activity of carbohydrate-responsive element-binding protein (ChREBP), which is a coactivator of lipogenic genes via the acetylation of Lys-672 in the development of NAFLD [17]. However, little is known about the relationship between HATs and the

\footnotetext{
This work was supported by the Main Research Program (E-0150301) of the Korea Food Research Institute (KFRI), funded by the Ministry of Science, ICT \& Future Planning.

${ }^{\S}$ Corresponding Author: Hyo-Kyoung Choi, Tel. 82-63-219-9421, Fax. 82-63-219-9876, Email. chkyoung@kfri.re.kr

Received: September 13, 2018, Revised: December 18, 2018, Accepted: February 12, 2019

This is an Open Access article distributed under the terms of the Creative Commons Attribution Non-Commercial License (http://creativecommons.org/licenses/by-nc/3.0/) which permits unrestricted non-commercial use, distribution, and reproduction in any medium, provided the original work is properly cited.
} 
development of NAFLD.

Unlike genetic changes, epigenetic modifications, like Lys acetylation, are potentially reversible and can be modified by environmental, dietary, and lifestyle factors [18]. Additionally, these epigenetic factors are closely associated with the phenotype and determine progression of NAFLD [19]. In this study, we investigated the correlation between HAT activity and protein acetylation in NAFLD and identified natural extracts (NExs) that attenuate lipid accumulation by modulating HAT activity in an NAFLD model of HepG2 cells.

\section{MATERIALS AND METHODS}

\section{Cell culture}

cells Human hepatocellular carcinoma (HepG2) cells were purchased from the American Type Culture Collection (Manassas, VA, USA) and cultured in a humidified atmosphere of $5 \% \mathrm{CO}_{2}$ at $37^{\circ} \mathrm{C}$ using high-glucose Dulbecco's modified Eagle's medium (DMEM) supplemented with $10 \%$ fetal bovine serum (FBS) and antibiotics (Welgene, Daegu, Republic of Korea). To establish an NAFLD model in HepG2 cells, a non-fat BSA-conjugated combination of oleic acid (OA) (Sigma-Aldrich, St. Louis, MO, USA) and palmitic acid (PA) (Sigma) was used at a ratio of 4:1 (OPA). HepG2 cells were simultaneously treated with $200 \mu \mathrm{g}$ of natural extracts and OPA for $24 \mathrm{~h}$.

\section{Preparation of natural extracts}

Based on 2015 Korea National Health and Nutrition Examination Survey, about 80 natural food which are frequently consumed by Korean chosen, and 22 natural food extracts among them in which experiments had been first finished were analyzed in

Table 1. Abbreviation of natural extracts used in this study.

\begin{tabular}{rllc}
\hline No. & \multicolumn{1}{c}{ Scientific name } & \multicolumn{1}{c}{ English name } & $\begin{array}{c}\text { Abbreviation used } \\
\text { in this study }\end{array}$ \\
\hline 1 & Allium tuberosum & Galic chives & EAT \\
2 & Morus alba & Mulberry & EMA \\
3 & Schizandra chinensis Bail & Magnolia vine & ESC \\
4 & Spinacia oleracea & Spinach & ESO \\
5 & Solanum lycopersicum & Tomato & ESL \\
6 & Citrus unshiu & Citrus & ECU \\
7 & Perilla frutescens & Perilla leaf & EPF \\
8 & Allium fistulosum & Green onion & EAF \\
9 & Lactuca sativa & Lettuce & ELS \\
10 & Allium cepa & Onion & EAC \\
11 & Codonopsis lanceolata & Doduk & ECL \\
12 & Cucurbita maxima & Sweet pumpkin & ECM \\
13 & Helianthus tuberosus & Jerusalem artichoke & EHT \\
14 & Zingiber officinale & Ginger & EZO \\
15 & Brassica oleracea & Broccoli & EBO \\
16 & Dioscorea polystachya & Hemp & EDP \\
17 & Capsicum annum & Bell pepper & ECA \\
18 & Platycodon grandiflorus & Bellflower & EPG \\
19 & Porphyra tenera & Laver & EPT \\
20 & Fragaria x ananassa & Strawberry & EFA \\
21 & Allium monanthum & Wild Chive & EAM \\
22 & Malus domestica & Apple & EMD \\
\hline & & & \\
\hline
\end{tabular}

the current study (Table 1). The natural food used in this study purchased from a local market (Seongnam, Gyeongggi-do, Korea). A voucher specimen has been deposited in Korea Food Research Institute (KFRI). Following cleaning of specimens those were extracted in a 10 -fold volume of $70 \%$ ethanol by shaking for $24 \mathrm{~h}$ at $25^{\circ} \mathrm{C}$. Finally, the precipitate was eliminated by centrifugation at $8,000 \times \mathrm{g}$ for $30 \mathrm{~min}$, and supernatants were lyophilized in a freeze- drier (II Shin, Dongdochum-Si, Korea).

\section{Cell toxicity}

Cells (approximately $5 \times 10^{4}$ ) were seeded in 24-well plates. After reaching approximately $70 \%$ confluence, cells were expos in OPA in presence or absence of NExs. After $24 \mathrm{~h}$ of incubation, $10 \mu \mathrm{L}$ of WST-1 solution (Enzo Life Sciences, Inc., Farmingdale, NY, USA) was added for $3 \mathrm{~h}$, and then absorbance of $100 \mu \mathrm{L}$ of the supernatant was measured at $450 \mathrm{~nm}$ (Molecular Devices, Sunnyvale, CA, USA).

\section{Oil red $O$ staining}

Cells (approximately $5 \times 10^{4} /$ well) were seeded. Reached approximately $70 \%$ confluence, cells were treated in OPA, alone or in combination with NExs at $200 \mu \mathrm{g} / \mathrm{mL}$. To measure lipid accumulation in HepG2 cells, we performed Oil Red O staining as previously described [20].

\section{HAT assay}

HeLa cell nuclear extract was purchased from Biovision Biotechnology (Milpitas, CA, USA). Nuclear extracts were assessed for HAT activity using a commercially available kit, according to the manufacturer's protocol (Biovision Biotechnology).

\section{Histone extraction}

In brief, $5 \times 10^{6}$ cells were seeded in 10-mm dishes. OPA was added for $18 \mathrm{~h}$ when the confluence reached approximately $70 \%\left(\sim 2 \times 10^{8}\right.$ cells). HepG2 cells were lysed according to the manufacturer's protocol (Abcam, Cambridge, MA, USA) pre-lysis buffer to isolate the nuclear fraction. The nuclear pellet was resuspended in lysis buffer, incubated for $30 \mathrm{~min}$ on ice, and centrifuged at $10,000 \mathrm{rpm}$ for $5 \mathrm{~min}$ at $4^{\circ} \mathrm{C}$. The supernatant was transferred to a new tube and DTT buffer was added. Samples were stored at $-70^{\circ} \mathrm{C}$ until analysis.

Table 2. Primers used in this study

\begin{tabular}{llll}
\hline & & \multicolumn{2}{c}{ Primer list } \\
\hline SREBP-1C & Forward & 5'-AAACTCAAGCAGGAGAACCTAAGTCT-3' & \\
& Reverse & 5'-GTCAGTGTGTCCTCCACCTCAGT-3' & \\
ACLY & Forward & 5'-TACCACCTCAGCCATCCAGA-3' & \\
& Reverse & 5'-GACCCCAACGAGACCAAGTT-3' & \\
FAS & Forward & 5'-AACCGGCTCTCCTTCTTCTTCGACTT-3' & qRT-PCR for \\
& Reverse & 5'-TCCGAGCGGCAGTACCCATTC-3' & \\
& Reverse & 5'-TGG AGA GGG ACT TGG GGT AG-3' & \\
PPAR & Forward & 5'-CCACCAACTTCGGAATCAGCT-3' & \\
& Reverse & 5'-TTTGGGATCCGGCAGTTAAGA-3' & \\
GAPDH & Forward & 5'-ATGTTCGTCATGGGTGTGAC-3' \\
& Reverse & 5'-GCATGGACTGTGGTCATGAGT-3' & \\
\hline
\end{tabular}

$S R E B P-1 C$, sterol regulatory element-binding protein-1c; $A C L Y$, ATP-citrate lyase $F A S$, Fatty acid synthase; PPAR- $\gamma$, peroxisome proliferator-activated receptor- $\gamma$; $G A P D H$, glyceraldehyde-3-phosphate dehydrogenase. 
Table 3. Antibodies used in this study

\begin{tabular}{|c|c|c|c|c|c|}
\hline & Vendor & Local & Country & Titer & Usage \\
\hline aН3К9ас & Cell signaling & Denvers, MA & USA & $1: 1000$ & \\
\hline aH4K8ac & Cell signaling & Denvers, MA & USA & $1: 1000$ & \\
\hline aH4K16ac & Cell signaling & Denvers, MA & USA & $1: 1000$ & \\
\hline aTotal Acetyl -Lys & Cell signaling & Denvers, MA & USA & $1: 1000$ & W. B \\
\hline aHDAC1 & Santa cruz biotechnology & Dallas, Texas & USA & 1:1000 & \\
\hline aßActin & Enzo life science & Farmingdale, NY & USA & 1:10000 & \\
\hline aaTubulin & Cell signaling & Denvers, MA & USA & $1: 5000$ & \\
\hline
\end{tabular}

HDAC: histone deacetylases

\section{Quantitative real-time $R T-P C R$}

Cells were seeded at $5 \times 10^{4}$ cells/well. After reaching approximately $70 \%$ confluence, cells were treated with or without OPA for $18 \mathrm{~h}$. To observe the effect of HATs on lipid accumulation in HepG2 cells, C-646, a p300/CBP-specific inhibitor, was added with OPA to HepG2 cells for $18 \mathrm{~h}$, and then we performed qRT-PCR as previously described [20]. The primers used in this study were listed up in Table 2.

\section{Immunoblotting}

Following treatment, cell extracts were supplemented with lysis buffer (Cell signaling) containing protease and phosphatase inhibitors (Roche, Basel, Switzerland) for $30 \mathrm{~min}$ and centrifugation at $20,000 \times \mathrm{g}$ for $20 \mathrm{~min}$ at $4^{\circ} \mathrm{C}$. The cell lysates were separated by SDS-PAGE and transferred to nitrocellulose membranes. The membranes were incubated with specific antibodies as indicated in Table 3 overnight at $4^{\circ} \mathrm{C}$, followed by incubation with a secondary antibody (Thermo Scientific, Rockford, IL, USA). Proteins were visualized using a ChemiDoc system (Bio-Rad) with an enhanced chemiluminescence substrate (Thermo Scientific).

\section{Statistical analysis}

qRT-PCR data were analyzed using $t$-tests. Correlations between HAT activity and lipid accumulation in HepG2 cells were evaluated by Pearson's correlation coefficients. Statistical analyses were carried out in SPSS (Ver. 20; SPSS Inc., Chicago, IL, USA). $P<0.05$ was considered statistically significant.

\section{RESULTS}

The acetylation status of non-histone and histone proteins increased by OPA

To examine whether the acetylation level of non-histone proteins and histone proteins increased in the NAFLD model, the acetylation status was evaluated in HepG2 cells with or without OPA treatment. In particular, cells were treated with $400 \mu \mathrm{M}$ of $\mathrm{OA}$ and $100 \mu \mathrm{M}$ of PA $(1 \times)$ or $800 \mu \mathrm{M}$ of $\mathrm{OA}$ and $200 \mu \mathrm{M}$ of PA (2X) with or without trichostatin A (TSA). As shown in Fig. $1 A$, the acetylation level of total proteins was higher in the samples treated with OPA and TSA than in the control group. Additionally, the acetylation level was relatively higher in the group treated with a high concentration of OPA. Next, to observe changes in the specific acetylation status of histone tails, H3K9, H4K8, and H4K16, related to the expression of genes related to lipid accumulation in liver, were extracted from HepG2 cells. Unlike total protein acetylation, H3K9, H4K8, and H4K16 were acetylated without TSA treatment and the acetylation levels increased in proportion to the concentration of OPA (Fig. 1B). Although the levels of acetylation at H3K9, H4K8, and

(A)

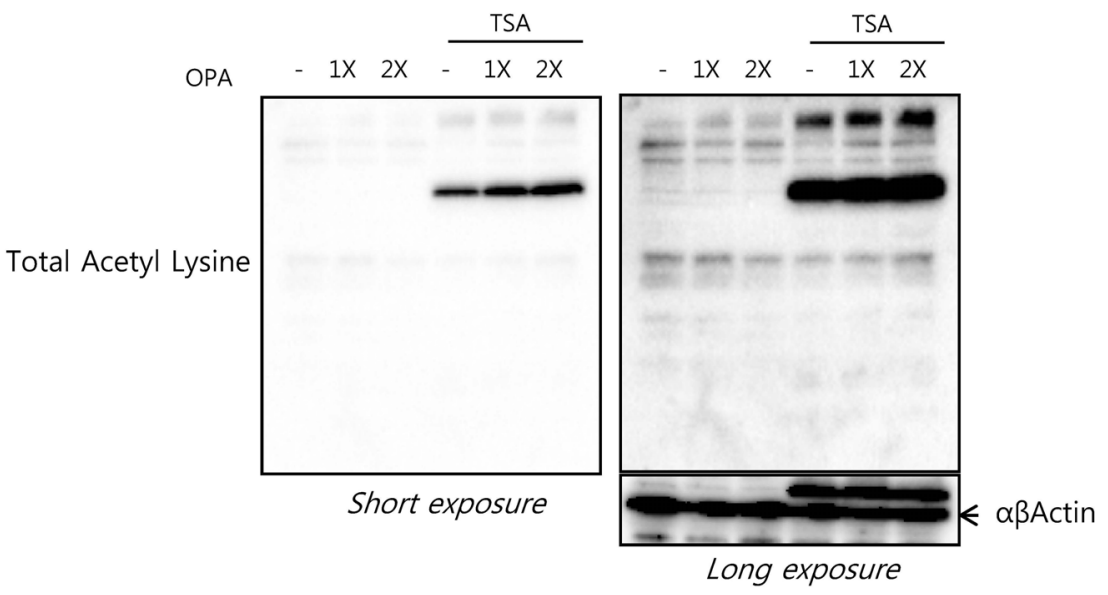

Fig. 1. Oleic and Palmitic acids (OPA)-induced activation of HAT increased non-histone and histone acetylation. (A), (B). Total acetylation of non-histone proteins and histone actylation were increased following OPA treatment. HepG2 cells were treated with either 1X or 2X OPA for 24 hr, in the presence or absence of TSA, HDAC inhibitor. Either total proteins or histone proteins were extracted from the cells, and the acetylation status was determined as the indicated antibodies. (B). (C). The HAT activity was measured in an NE from HepG2 cells. Activity in the OPA-treated group is presented as a percentage relative to that in the control group, which was incubated without OPA. The values presented are the means \pm SD of three independent experiments. * $P<0.05$ (left panel). To confirm nuclear fraction, western blotting was carried out. HDAC1 and tubulin were used as nuclear and cytosolic markers, respectively (right panel). 1XOPA, $400 \mu \mathrm{M}$ OA+100 $\mu \mathrm{M}$ PA; 2XOPA, $800 \mu \mathrm{M} O \mathrm{OA}+200 \mu \mathrm{M}$ PA; TSA, trichostatin A; HDAC, histone deacetylases. 
(B)

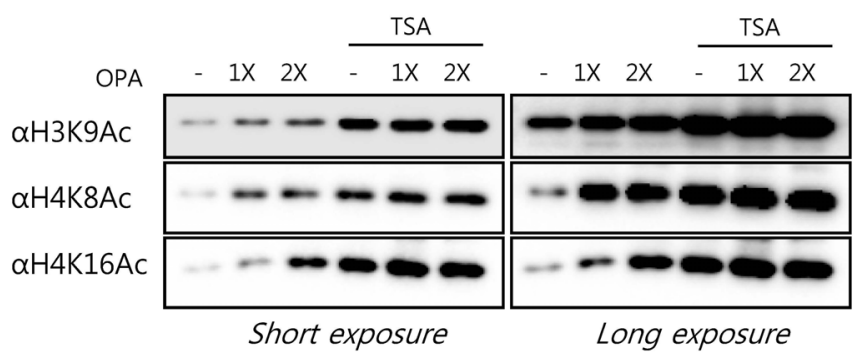

(C)

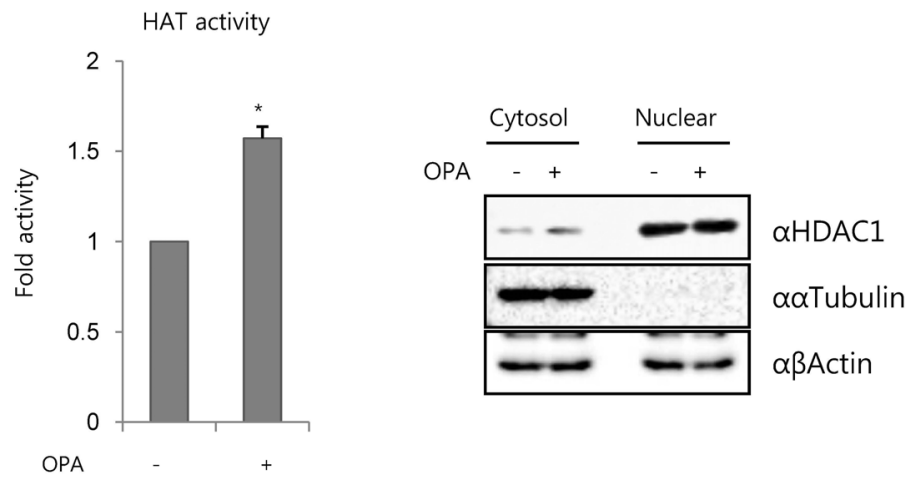

Fig. 1. continued

(A)

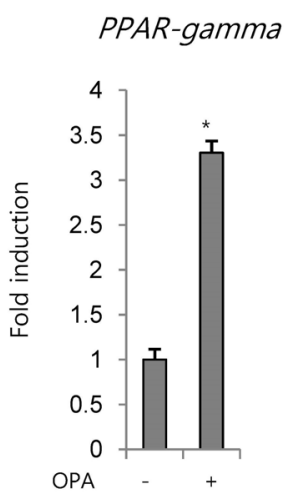

(B)

\section{PPAR-gamma}

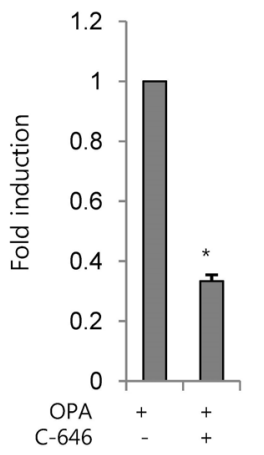

SREBP1C

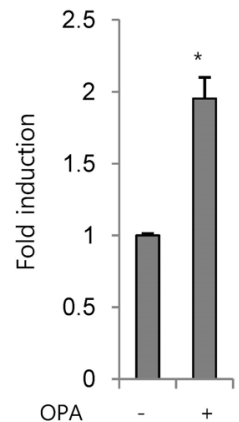

SREBPIC

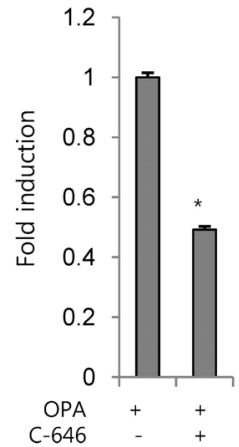

$A C L Y$

FASN
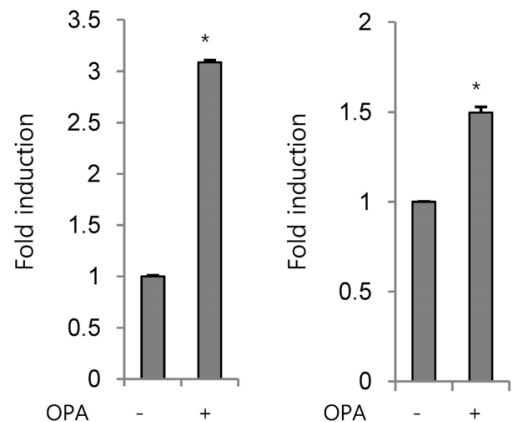

FASN

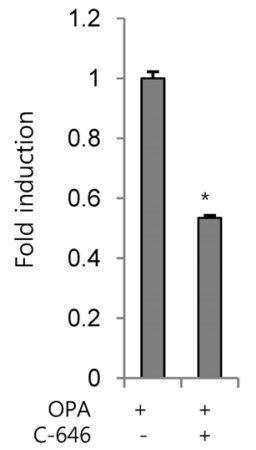

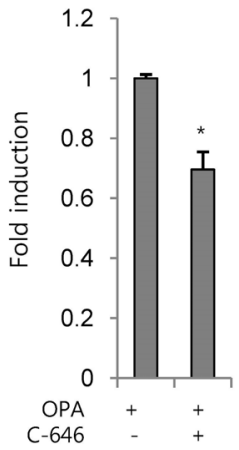

Fig. 2. mRNA expression of lipogenic genes is dependent on HAT activity. (A) mRNA expression of genes related lipid metabolism was measured by qRT-PCR. Oleic and palmitic acid (OPA) was treated for $24 \mathrm{hr}$ in HepG2 cells, RNA was extracted, and then $2 \mu \mathrm{g}$ RNA was synthased to cDNA All experiments were carried out three times independently and data are expressed as mean $\pm \mathrm{SD}$. * $P<0,05$ (student t-test). (B) C-646, a specific inhibitor of p300/CBP, blocked the OPA-induced mRNA expression of lipogenic genes. HepG2 cells were treated with OPA in the presence or absence of C-646 for $18 \mathrm{~h}$. mRNA expression of $A C L Y, F A S N$, SREBP-1C, and PPAR, was measured by qRT-PCR. The values presented are the means $\pm S D$ of three independent experiments. Data are expressed as mean $\pm S D$. ${ }^{\star} P<0.05$ (student t-test). PPAR- $\gamma$, peroxisome proliferator-activated receptor- $\gamma$; $S R E B P 1 C$, sterol regulatory element-binding protein-1c; $A C L Y$, ATP citrate lyase; FASN, fatty acid synthase. 
H4K16 increased in the groups treated with both OPA and TSA, similar to the patterns observed for the groups treated with OPA only, the degree of acetylation was much greater than that of groups treated with OPA only (Fig. 1B). Based on the close association between protein acetylation and HAT activity, we investigated whether HAT activity was greater in the NAFLD model than in the control. As expected, HAT activity was much greater in the group treated with OPA (Fig. 1C). Taken altogether, these results showed that the acetylation levels of both non-histone and histone proteins were positively related to HAT activity, which increased by lipid accumulation in HepG2 cells.
Lipogenic gene expression decreased by blocking HAT activity using C-646, a p300/CBP-specific inhibitor

We next investigate the effect of OPA on the expression of lipogenesis-related genes, such as peroxisome proliferatoractivated receptor- $\gamma(P P A R \gamma)$, sterol regulatory element-binding protein-1c (SREBP1C), ATP citrate lyase(ACLY), and fatty acid synthase $(F A S N)$, in HepG2 cells. Consistent with the results of previous studies, we found that an increase in lipogenic gene expression was related to lipid accumulation (Fig. 2A). To elucidate the relationship between the mRNA expression of lipogenic genes and HAT activity, we incubated HepG2 cells

(A)
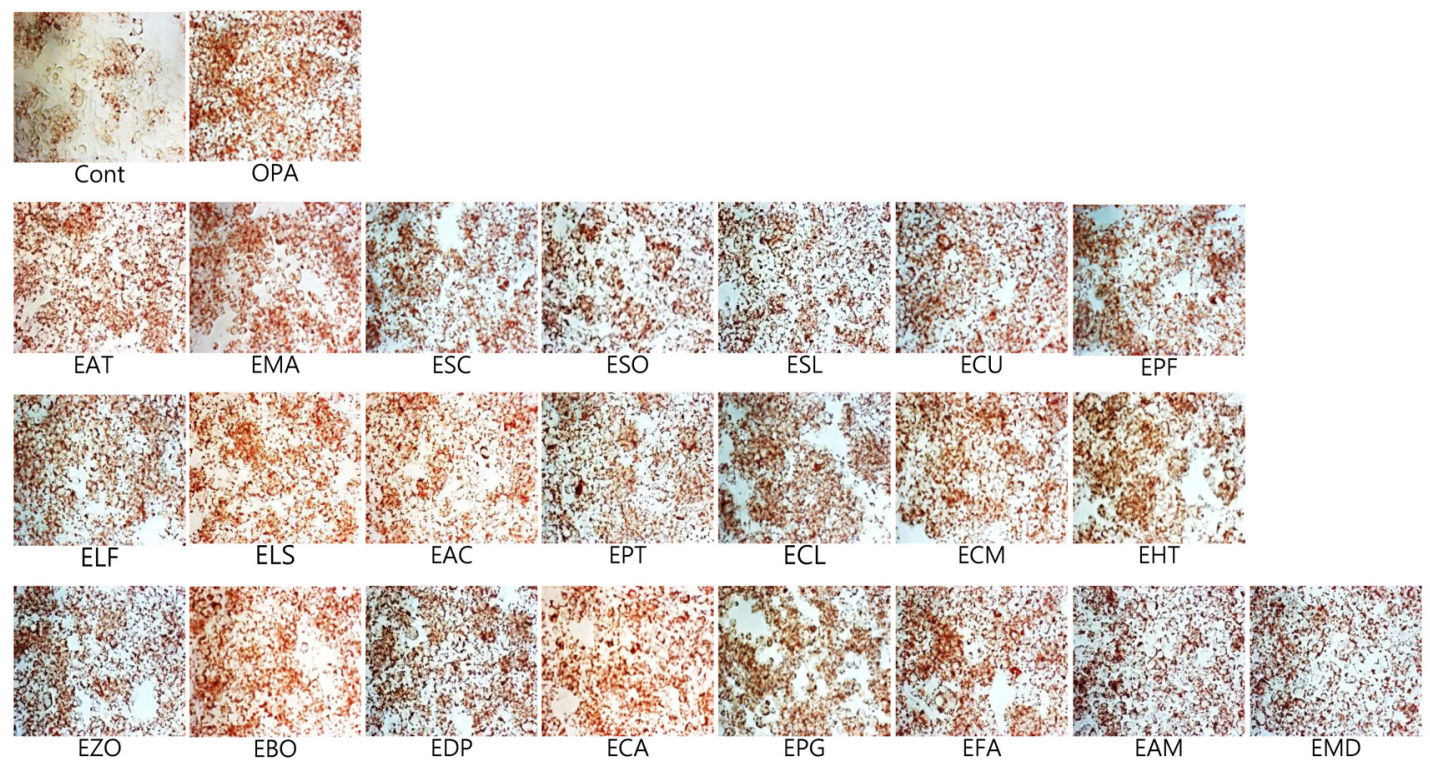

(B)
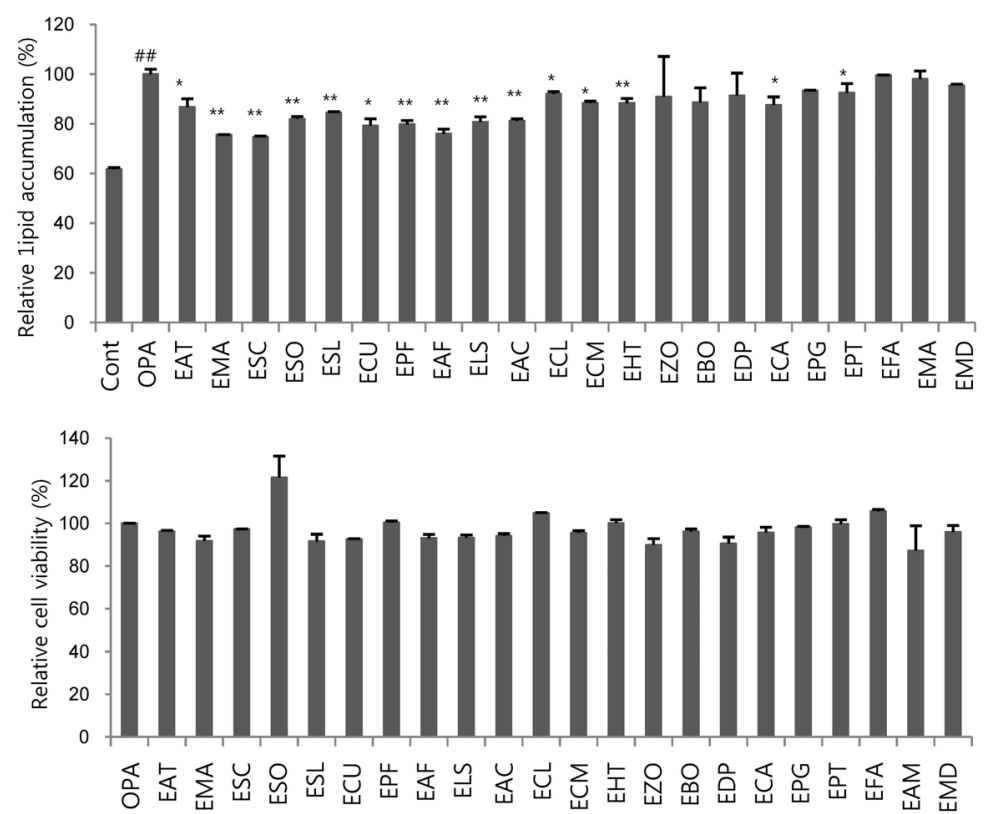

Fig. 3. Effect of natural extracts (NExs) on lipid accumulation in HepG2 cells. (A) HepG2 cells were treated oleic acid only or co-treated with oleic acid and NExs for $24 \mathrm{~h}$. Cells were stained with Oil red $O$ staining as described in Materials and Methods and were viewed by microscopy (upper panel). Quantitative Oil red O dye as fat drops and data are expressed as mean $\pm \mathrm{SD}$. \#\# control group, ${ }^{\star *} P<0.01,{ }^{\star} P<0.05$ (student t-test) (low panel). (B) Cytotoxicity test of NExz in HepG2 cells. HepG2 cells were treated with the various NExs in the presence or absence of oleic and palmitic acids (OPA) for $24 \mathrm{~h}$. The cell viability was measured using a WST-1 assay in HepG2 cells. All data are expressed as mean \pm SD from the samples of each group. There was no statistical significance. 


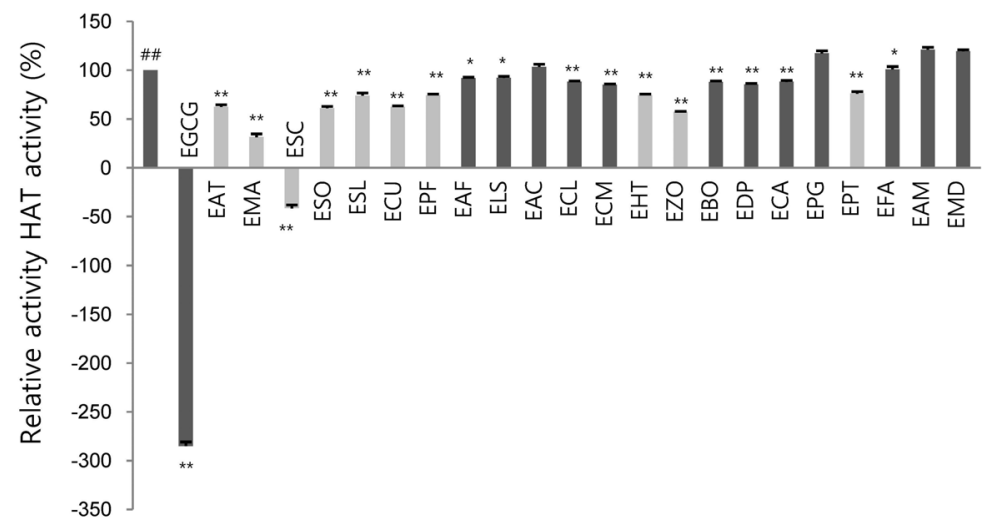

Fig. 4. Effect of natural extracts (NExs) on histone acetyl transferase activity. (A) HAT activity assays were performed with NExs as described in Materials and Methods. The result was represented with relative percentage compared to a positive control which was incubated without a NEx. Values are means \pm SD for three independent experiments. \#\# control group, ${ }^{* \star} P<0.01,{ }^{\star} P<0.05$ (student t-test). Epigallocatechin gallate (EGCG) was used as a negative control.

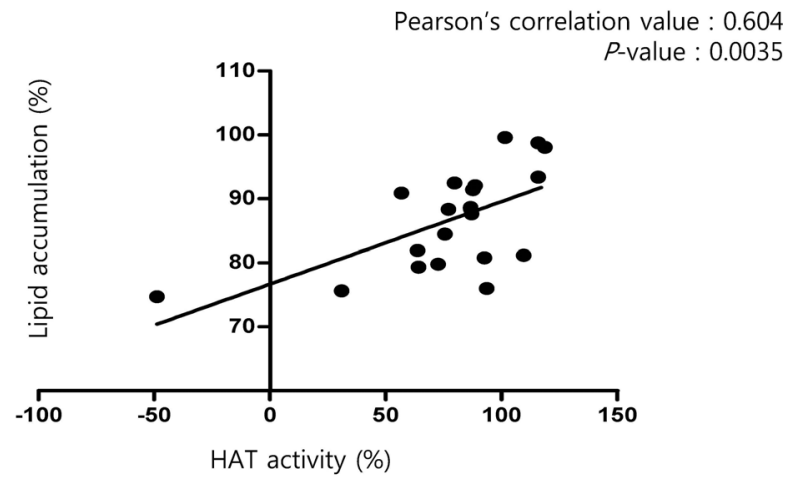

Fig. 5. There are positive correlation between lipid accumulation and HAT activity. To validate the correlation between HAT activity and lipid accumulation, Pearson's correlation value was calculated based on the experimental data from Oil red O (Fig. 3A) and HAT assay (Fig. 4) following NExs treatment with oleic and palmitic acids (OPA). Pearson's correlation value is 0.604 .

with or without C-646, a specific p300/CBP inhibitor, in combination with OPA for $18 \mathrm{~h}$. As shown in Fig. 2B, the mRNA expression of lipogenic genes, i.e., PPAR, SREBP1C, $A C L Y$, and FASN, was abrogated by blocking p300/CBP activity using C-646. Collectively, our data suggest that there is a correlation between the expression of lipogenic genes and HAT activity.

\section{EMA and ESC effectively prevent OPA-induced lipid accumulation} in HepG2 cells

We hypothesized that natural compounds that can block OPA-induced lipid accumulation may show relatively high anti-HAT activity. Thus, we selected 22 commonly consumed vegetables in Korea and measured their ability to prevent OPAinduced lipid accumulation in HepG2 cells. $200 \mu \mathrm{g} / \mathrm{mL}$ extract with OPA were treated in cells, and lipid accumulation was measured by Oil Red $O$ staining (Fig. 3A, upper panel). To quantify it, absorbance of the dissolved Oli Red $O$ dye in isopropanol was measured (Fig. 3A, lower panel). Lipid accumulation was greater in OPA-treated cells than in the control group, and most extracts, including the extract of Allium tuberosum (EAF), had a protective effect on lipid accumulation. In particular, the extract of Morus alba (EMA) and that of
Schizandra chinensis Bail (ESC) reduced lipid accumulation by almost $30 \%$ compared to that in cells treated with OPA alone. The extracts of Platycodon grandiflorus, Fragaria $x$ ananassa, Allium monanthum, and Malus domestica did not have preventive effects on OPA-induced lipid accumulation in HepG2 cells. We examine whether the effect of NExs on the inhibition of lipid accumulation occurred as cytotoxicity. HepG2 cells were incubated in the presence or absence of OPA, with or without NExs. As shown in Fig. 3B, NExs did not influence the viability of cells. Altogether, NExs used in this study significantly abrogates OPA-induced lipid accumulation without cytotoxicity.

ESC shows the strongest effect on HAT activity

To analyze the correlation between lipid accumulation and HAT activity, we used a cell-free system. NEs at the same concentrations used in the previous experiment were incubated with HeLa nuclear extract, and epigallocatechin-3-gallate (EGCG), which is a well-established specific HATi [21], was used for comparison. As shown in Fig. 4, anti-HAT activity was observed in $22 \mathrm{NEs}$. Among these, $10 \mathrm{NEs}$, i.e., EAT, EMA, ESC, ESO, ESL, ECU, EPF, EHT, EZO, and EPT, inhibited HAT activity by over $20 \%$ in comparison with activity in the control group (gray bars in Fig. 4). ESC demonstrated the strongest anti-HAT effect, and EMA also showed a relatively strong inhibitory effect, supporting the correlation between lipid accumulation and HAT activity.

Lipid accumulation and HAT activity are positively correlated

To further evaluate the correlation between lipid accumulation and HAT activity, Pearson's correlation coefficients were calculated using Oil Red $\mathrm{O}$ absorbance data and HAT activity for each NE (Fig. 5). The correlation coefficient was 0.604 ( $P$ $=0.0035)$, suggesting that there is a positive correlation between lipid accumulation and HAT activity.

\section{DISCUSSION}

NAFLD is the most common liver disease worldwide. Many studies have focused on the effects of natural dietary compounds on lipid metabolism to develop new therapeutic strategies [22]. However, lifestyle influences the development 
and progression of NAFLD via epigenetic regulation [23], since it is a complex disease determined, in part, by environment factors [24]. Thus, epigenetic mechanisms involved in lipid metabolism are regarded as potential preventive or therapeutic targets [25].

Our data supported the results of previous studies showing that NAFLD may be controlled by epigenetic regulatory mechanisms. In particular, the balance between histone acetylation/ deacetylation at the N-termini, which are catalyzed by HAT and HDAC, respectively [26], influences gene expression profiles in NAFLD [27]. As shown in Fig. 1B, histone H3K9, H4K8, and H4K16 acetylation increased dramatically following OPA treatment, and the acetylation status increased when HDAC activity was inhibited by TSA, an HDAC-specific inhibitor, indicating that aberrant histone acetylation by an imbalance between HAT and HDAC activity is involved in the hyperacetylation of histones, resulting in the development and progression of NAFLD. In adipocytes, histone H3K56 acetylation was first identified in a genome-wide profile analysis [28]. Mikula et al. demonstrated that the acetylation of histones $\mathrm{H} 3 \mathrm{~K} 9$ and H3K18 in the TNFa and $\mathrm{CCl} 2$ promoters was increased in the livers of mice fed a high-fat diet [29]. Furthermore, our results indicated that the total protein acetylation status increased significantly in cells treated with OPA in combination with TSA. Unexpectedly, acetylated-Lys was not detected without HDAC inhibition in extracts of the total cell lysate, unlike that of histones (Fig. 1A), suggesting that HDACs, which are predominantly localized in the cytosol or shuttle between the cytoplasm and the nucleus, are closely involved in the regulation of acetylation on nonhistone proteins related to lipid accumulation. Similarly, a previous study reported that total protein acetylation is significantly increased in the hearts of HFD-fed mice [30], suggesting that lipid accumulation-induced hyperacetylation is not limited to the liver and is involved in obesity-related diseases in various organs. In particular, SREBP1c is acetylated by $\mathrm{p} 300 / \mathrm{CBP}$ in both cells and mouse livers, leading to increased stability and recruitment to its lipogenic target gene promoter [31]. p300/CBP hyperactivity was also associated with ChREBP acetylation and hepatic steatosis in a mouse model of obesity [17]. Accordingly, the increase in HAT activity in OPA-treated cells (Fig. 1C) was expected.

Transcription is a highly regulated process, and acetylation plays a major role in this regulation [32] by the various conformational changes of the chromatin, including charge neutralization [33], consequently providing unique biding surfaces for activators of transcription [34]. PPARy and SREBP1c regulate genes required for lipid metabolism, such as ACLY or FASN [35]. The mRNA expression of PPAR $\gamma$ is up-regulated in the liver of obese patients with NAFLD, reinforcing the lipogenic mechanism in response to SREBP-1C induction in the development of NAFLD [36]. Considering previous studies, our data (Fig. 2A) showing the increase in lipogenic gene expression is not unexpected. However, the reduction in lipogenesis-related gene transcription following C-646 treatment was noteworthy, indicating that dynamic histone acetylation and deacetylation are also involved in the transcriptional activation of lipogenesisrelated genes.

Currently, there are no approved pharmacological agents or
FDA guidelines for the treatment of NAFLD [37]. Anti-obesity drugs, such as orlistat and sibutramine, are used for NAFLD but have potentially hazardous side effects [38]. Accordingly, a variety of natural phytochemical compounds to prevent NAFLD have been explored. In our study, 15 of 22 NExs prevented OPA-induced lipid accumulation in HepG2 cells (Fig. 3A). The anti-lipogenic effects of the extracts, including EMA, ESC, and EFA, are well established $[39,40]$. However, our aim was to elucidate the mechanisms involved in the beneficial effects of NExs on NAFLD prevention from a new perspective, i.e., epigenetics. Alterations in acetylation status associated with chronic metabolic diseases, including the abnormal regulation of hepatic lipid metabolism, have been related in the development and progression of NAFLD [2]. Certain bioactive foods may have a beneficial effect on NAFLD via their effects on acetylation. Interestingly, two NExs, EMA and ESC, demonstrated strong anti-lipogenic effects and inhibited HAT activity (Fig. 4), suggested that there is a positive correlation between lipid accumulation and HAT activity. An analysis of the correlation between these two factors supported this finding. Although the relationship was not strong (Fig. 5, Pearson's correlation coefficient $=0.604$ ), it was sufficient to suggest a new approach for the prevention or treatment of NAFLD based on natural foods with epigenetic effects.

The present study had many limitations. The number of preventative natural food extracts examined in the study was small. To definitively establish the correlation between lipid accumulation and HAT activity, further studies of the molecular mechanism are needed.

\section{AVAILABILITY OF DATA AND MATERIAL}

All data are contained and described within the manuscript.

\section{AUTHORS' CONTRIBUTIONS}

H.-K.C. conceived and designed the experiment and wrote the manuscript. S.-W.C. performed the experiments and wrote a draft of the manuscript. J.-T.H. and J.-H.P supported the experiments. H.-K.C supervised the work and critically reviewed the manuscript.

\section{CONFLICT OF INTERESTS}

The authors declare no potential conflicts of interests.

\section{ORCID}

Sangwon Chung: https://orcid.org/0000-0001-7773-2195

Jin-Taek Hwang: https://orcid.org/0000-0002-6650-7934

Jae Ho Park: https://orcid.org/0000-0003-4428-436X

Hyo-Kyoung Choi: https://orcid.org/0000-0001-9424-0432

\section{REFERENCES}

1. Ahmed MH, Husain NE, Almobarak AO. Nonalcoholic Fatty liver disease and risk of diabetes and cardiovascular disease: what is important for primary care physicians? J Family Med Prim Care 
2015;4:45-52.

2. Sun C, Fan JG, Qiao L. Potential epigenetic mechanism in non-alcoholic fatty liver disease. Int J Mol Sci 2015;16:5161-79.

3. Wang Q, Guo S, Gao Y. Protein lysine acetylated/deacetylated enzymes and the metabolism-related diseases. Adv Biosci Biotechnol 2016;7:454-67.

4. Castaño-Cerezo S, Bernal V, Post H, Fuhrer T, Cappadona S, Sánchez-Díaz NC, Sauer U, Heck AJ, Altelaar AF, Cánovas M. Protein acetylation affects acetate metabolism, motility and acid stress response in Escherichia coli. Mol Syst Biol 2014;10:762.

5. van Noort V, Seebacher J, Bader S, Mohammed S, Vonkova I, Betts MJ, Kühner S, Kumar R, Maier T, O'Flaherty M, Rybin V, Schmeisky A, Yus E, Stülke J, Serrano L, Russell RB, Heck AJ, Bork P, Gavin AC. Cross-talk between phosphorylation and lysine acetylation in a genome-reduced bacterium. Mol Syst Biol 2012;8:571.

6. Yu BJ, Kim JA, Moon JH, Ryu SE, Pan JG. The diversity of lysine-acetylated proteins in Escherichia coli. J Microbiol Biotechnol 2008;18:1529-36.

7. Kim D, Yu BJ, Kim JA, Lee YJ, Choi SG, Kang S, Pan JG. The acetylproteome of Gram-positive model bacterium Bacillus subtilis. Proteomics 2013;13:1726-36.

8. Fukushima A, Lopaschuk GD. Acetylation control of cardiac fatty acid $\beta$-oxidation and energy metabolism in obesity, diabetes, and heart failure. Biochim Biophys Acta 2016;1862:2211-20.

9. LaBarge S, Migdal C, Schenk S. Is acetylation a metabolic rheostat that regulates skeletal muscle insulin action? Mol Cells 2015;38: 297-303.

10. Clayton AL, Hazzalin CA, Mahadevan LC. Enhanced histone acetylation and transcription: a dynamic perspective. Mol Cell 2006;23:289-96.

11. Yang XJ, Seto E. The Rpd3/Hda1 family of lysine deacetylases: from bacteria and yeast to mice and men. Nat Rev Mol Cell Biol 2008;9:206-18.

12. Haberland M, Montgomery RL, Olson EN. The many roles of histone deacetylases in development and physiology: implications for disease and therapy. Nat Rev Genet 2009;10:32-42.

13. Zhao $S, X u$ W, Jiang $W, Y u$ W, Lin $Y$, Zhang $T$, Yao J, Zhou L, Zeng Y, Li H, Li Y, Shi J, An W, Hancock SM, He F, Qin L, Chin J, Yang $\mathrm{P}$, Chen $\mathrm{X}$, Lei $\mathrm{Q}$, Xiong $\mathrm{Y}$, Guan KL. Regulation of cellular metabolism by protein lysine acetylation. Science 2010;327:1000-4.

14. Wang $Q$, Zhang $Y$, Yang C, Xiong $H$, Lin $Y$, Yao J, Li H, Xie L, Zhao W, Yao Y, Ning ZB, Zeng R, Xiong Y, Guan KL, Zhao S, Zhao GP. Acetylation of metabolic enzymes coordinates carbon source utilization and metabolic flux. Science 2010;327:1004-7.

15. Podrini C, Borghesan M, Greco A, Pazienza V, Mazzoccoli G, Vinciguerra M. Redox homeostasis and epigenetics in non-alcoholic fatty liver disease (NAFLD). Curr Pharm Des 2013;19:2737-46.

16. Xu X, So JS, Park JG, Lee AH. Transcriptional control of hepatic lipid metabolism by SREBP and ChREBP. Semin Liver Dis 2013;33:301-11.

17. Bricambert J, Miranda J, Benhamed F, Girard J, Postic C, Dentin R. Salt-inducible kinase 2 links transcriptional coactivator p300 phosphorylation to the prevention of ChREBP-dependent hepatic steatosis in mice. J Clin Invest 2010;120:4316-31.

18. Shankar E, Kanwal R, Candamo M, Gupta S. Dietary phytochemicals as epigenetic modifiers in cancer: promise and challenges. Semin Cancer Biol 2016;40-41:82-99.

19. Chalasani N, Guo X, Loomba R, Goodarzi MO, Haritunians T, Kwon S, Cui J, Taylor KD, Wilson L, Cummings OW, Chen YD, Rotter J;
Nonalcoholic Steatohepatitis Clinical Research Network. Genomewide association study identifies variants associated with histologic features of nonalcoholic Fatty liver disease. Gastroenterology 2010;139:1567-76, 1576.e1-6.

20. Hwang JT, Shin EJ, Chung MY, Park JH, Chung S, Choi HK. Ethanol extract of Allium fistulosum inhibits development of non-alcoholic fatty liver disease. Nutr Res Pract 2018;12:110-7.

21. Choi KC, Jung MG, Lee YH, Yoon JC, Kwon SH, Kang HB, Kim MJ, Cha JH, Kim YJ, Jun WJ, Lee JM, Yoon HG. Epigallocatechin-3-gallate, a histone acetyltransferase inhibitor, inhibits EBV-induced $B$ lymphocyte transformation via suppression of RelA acetylation. Cancer Res 2009;69:583-92.

22. Pan MH, Lai CS, Tsai ML, Ho CT. Chemoprevention of nonalcoholic fatty liver disease by dietary natural compounds. Mol Nutr Food Res 2014;58:147-71.

23. Pirola CJ, Gianotti TF, Burgueño AL, Rey-Funes M, Loidl CF, Mallardi P, Martino JS, Castaño GO, Sookoian S. Epigenetic modification of liver mitochondrial DNA is associated with histological severity of nonalcoholic fatty liver disease. Gut 2013;62:1356-63.

24. Anstee QM, Day CP. The genetics of NAFLD. Nat Rev Gastroenterol Hepatol 2013;10:645-55.

25. Afonso MB, Rodrigues PM, Simão AL, Castro RE. Circulating microRNAs as potential biomarkers in non-alcoholic fatty liver disease and hepatocellular carcinoma. J Clin Med 2016;5:30.

26. Granger A, Abdullah I, Huebner F, Stout A, Wang T, Huebner T, Epstein JA, Gruber PJ. Histone deacetylase inhibition reduces myocardial ischemia-reperfusion injury in mice. FASEB J 2008;22: 3549-60.

27. Tian Y, Wong VW, Chan HL, Cheng AS. Epigenetic regulation of hepatocellular carcinoma in non-alcoholic fatty liver disease. Semin Cancer Biol 2013;23:471-82.

28. Lo KA, Bauchmann MK, Baumann AP, Donahue CJ, Thiede MA, Hayes LS, des Etages SA, Fraenkel E. Genome-wide profiling of H3K56 acetylation and transcription factor binding sites in human adipocytes. PLoS One 2011;6:e19778.

29. Mikula M, Majewska A, Ledwon JK, Dzwonek A, Ostrowski J. Obesity increases histone $\mathrm{H} 3$ lysine 9 and 18 acetylation at Tnfa and $\mathrm{Ccl} 2$ genes in mouse liver. Int $J$ Mol Med 2014;34:1647-54.

30. Alrob OA, Sankaralingam S, Ma C, Wagg CS, Fillmore N, Jaswal JS, Sack MN, Lehner R, Gupta MP, Michelakis ED, Padwal RS, Johnstone $\mathrm{DE}$, Sharma AM, Lopaschuk GD. Obesity-induced lysine acetylation increases cardiac fatty acid oxidation and impairs insulin signalling. Cardiovasc Res 2014;103:485-97.

31. Ponugoti B, Kim DH, Xiao Z, Smith Z, Miao J, Zang M, Wu SY, Chiang CM, Veenstra TD, Kemper JK. SIRT1 deacetylates and inhibits SREBP-1C activity in regulation of hepatic lipid metabolism. J Biol Chem 2010;285:33959-70.

32. Sterner DE, Berger SL. Acetylation of histones and transcriptionrelated factors. Microbiol Mol Biol Rev 2000;64:435-59.

33. Verdone L, Agricola E, Caserta M, Di Mauro E. Histone acetylation in gene regulation. Brief Funct Genomics Proteomics 2006;5:209-21.

34. Kurdistani SK, Grunstein M. Histone acetylation and deacetylation in yeast. Nat Rev Mol Cell Biol 2003;4:276-84.

35. Fajas L, Schoonjans K, Gelman L, Kim JB, Najib J, Martin G, Fruchart JC, Briggs M, Spiegelman BM, Auwerx J. Regulation of peroxisome proliferator-activated receptor gamma expression by adipocyte differentiation and determination factor $1 /$ sterol regulatory element binding protein 1: implications for adipocyte differentiation and 
metabolism. Mol Cell Biol 1999;19:5495-503.

36. Pettinelli $P$, Videla LA. Up-regulation of PPAR-gamma mRNA expression in the liver of obese patients: an additional reinforcing lipogenic mechanism to SREBP-1c induction. J Clin Endocrinol Metab 2011;96:1424-30.

37. Tolman KG, Dalpiaz AS. Treatment of non-alcoholic fatty liver disease. Ther Clin Risk Manag 2007;3:1153-63.

38. Rodgers RJ, Tschöp MH, Wilding JP. Anti-obesity drugs: past, present and future. Dis Model Mech 2012;5:621-6.

39. Ann JY, Eo H, Lim Y. Mulberry leaves (Morus alba L.) ameliorate obesity-induced hepatic lipogenesis, fibrosis, and oxidative stress in high-fat diet-fed mice. Genes Nutr 2015;10:46.

40. Sun JH, Liu X, Cong LX, Li H, Zhang CY, Chen JG, Wang CM. Metabolomics study of the therapeutic mechanism of Schisandra Chinensis lignans in diet-induced hyperlipidemia mice. Lipids Health Dis 2017; 16:145. 